\title{
Interface states in photonic crystal heterostructures
}

\author{
Lan-Lan Lin \\ Institute of Physics, Chinese Academy of Sciences, P.O. Box 603, Beijing 100080, China
}

Zhi-Yuan Li

Department of Chemistry, University of Washington, Box 351700, Seattle, Washington 98195

and Department of Physics, Hong Kong University of Science and Technology, Clear Water Bay, Kowloon, Hong Kong, China

(Received 2 February 2000; revised manuscript received 7 August 2000; published 2 January 2001)

\begin{abstract}
We study the interface states formed in a heterostructure composed of two semi-infinite two-dimensional photonic crystals with different filling fractions. Photonic band structure calculations by means of a supercell technique show that localized interface states are absent in this simple heterostructure; they can be created either by lateral lattice slipping or by increasing the interface separation.
\end{abstract}

DOI: $10.1103 /$ PhysRevB.63.033310

PACS number(s): 68.65.-k, 42.79.Gn, 41.20.Jb

Photonic crystals are artificial periodic structures that have attracted extensive research interest in the last decade. ${ }^{1-3}$ As in conventional crystals, the Bragg scattering of electromagnetic (EM) waves will give rise to photonic band structures and band gaps. The existence of photonic band gaps may bring about some unusual physical phenomena such as inhibition of spontaneous emission ${ }^{1,4}$ with potential applications in wide scientific and technical areas. ${ }^{1-3}$

One of the promising applications of a photonic crystal is in molding the flow of light in some defect structures introduced in an otherwise perfect lattice. The characteristic of a highly-efficient photonic crystal waveguide is well established both theoretically and experimentally, ${ }^{5-10}$ and this waveguide is expected to hold the key to future all-optical circuits. So far, the photonic crystal waveguide has been created inside an infinite lattice as a line defect by removal of one or several rows of atoms. The waveguide can equivalently be assumed as an interface between two identical semi-infinite lattices, which are of the same lattice type, size, atom shape, and crystalline directions. The interface can, in principle, form between two different lattices with different physical or geometrical parameters, which can be called a heterostructure, similar to those in semiconductor physics. This interface is very common in realistic three-dimensional photonic crystals in optical wavelengths, which, because of the limits of modern microfabrication techniques, are essentially polycrystalline structures composed of many small single-crystalline grains. For example, there appear many cracks and dislocations in a typical inverse-opal photonic crystal. ${ }^{11}$ On the other hand, there is a long-standing interest in the states at a photonic crystal surface. ${ }^{12-14}$ This surface is an extreme example of a heterostructure, composed of one semi-infinite photonic crystal and one semi-infinite homogeneous medium. It is therefore interesting to see what happens to the interface state between two different photonic crystals.

In this report, we confine ourselves to a simple heterostructure consisting of two different two-dimensional (2D) semi-infinite lattices. As schematically shown by Fig. 1, the two lattices are both composed of square lattices of dielectric cylinders in a homogeneous background medium, and the interface is parallel to the lattice axis [(01) direction]. The only difference in geometrical and physical parameters of the two lattices is the filling fraction of cylinders, denoted as $f_{1}$ and $f_{2}$, respectively. In the simplest case, all cylinders lie exactly at the sites of an infinite square lattice. In addition, the two crystals can be slipped relative to each other in both longitudinal (01) and perpendicular (10) directions, generating two other kinds of more complex heterostructures. These heterostructures can be readily fabricated by the microlithography technique. One can also assume that a structure is formed by the touching of two photonic crystal samples.

The photonic problem for this $2 \mathrm{D}$ heterostructure can be solved numerically by the plane-wave expansion method in combination with a supercell technique. The supercell technique has often been utilized to investigate waveguide structures ${ }^{5-7}$ disordered systems, ${ }^{15}$ and surface waves ${ }^{12-14}$ in photonic crystals. In our numerical scheme, we consider the following wave equation satisfied by the magnetic field $\mathbf{H}(\mathbf{r})^{16,17}$

$$
\boldsymbol{\nabla} \times\left[\frac{1}{\boldsymbol{\epsilon}(\mathbf{r})} \boldsymbol{\nabla} \times \mathbf{H}(\mathbf{r})\right]=\frac{\omega^{2}}{c^{2}} \mathbf{H}(\mathbf{r}),
$$

where $\epsilon(\mathbf{r})$ is the periodic dielectric function of the 2D lattice. The photonic band structures for 2D photonic crystals in the TM (with the electric field along the cylinder axis) and TE (with the magnetic field along the cylinder axis) polarization modes can be solved based on this formula with the use of the plane-wave expansion method. ${ }^{16-19}$

The heterostructure is essentially aperiodic; however, as a good approximation, we can still use the plane-wave expansion method provided that it is combined with a supercell technique. The supercell should be large enough to guarantee that the coupling effect between neighboring supercells is negligible. For the heterostructure considered here, the supercell is a rectangle containing $m \times 1$ unit cells in each of the two lattices. In our calculations, we take $m=4$. Then the primary vectors of this supercell are $\mathbf{a}_{1}=(2 m, 0) a$ and $\mathbf{a}_{2}$ $=(0,1) a$, where $a$ is the lattice constant of the original square lattices. The corresponding reciprocal vectors are $\mathbf{b}_{1}$ $=(1 / 2 m, 0) 2 \pi / a$ and $\mathbf{b}_{2}=(0,1) 2 \pi / a$. The first Brillouin zone is also a rectangle. The four high-symmetry points are $\Gamma=(0,0), \quad X_{1}=(1 / 2 m, 0) \pi / a, \quad X_{2}=(0,1) \pi / a, \quad$ and $\quad M$ $=(1 / 2 m, 1) \pi / a$. The photonic band gap of the heterostruc- 


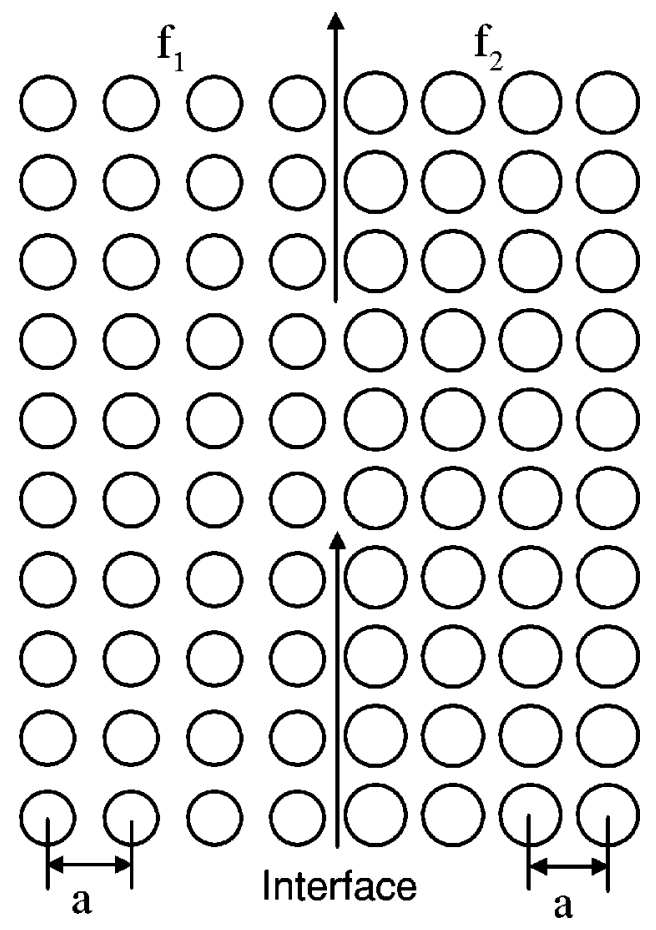

FIG. 1. Schematic configuration of heterostructure formed by two semi-infinite square lattices with different filling fractions of cylinders.

ture is determined by calculation of the photon density of states (DOS), which is performed only in the irreducible Brillouin zone of the supercell lattice.

Our calculation starts from the simplest heterostructure where all the cylinders lie in the sites of a square lattice. We first consider structures composed of dielectric cylinders in air. According to previous photonic band structure calculations, ${ }^{1,18-20}$ such a crystal structure has two significant band gaps between the 1-2 bands and the 3-4 bands in the TM mode. At a refractive index of the cylinder of $n=3.6$, the ground band gap lies at the frequency of $(0.228-$ $0.310)(2 \pi c / a)$ and $(0.219-0.283)(2 \pi c / a)$ for filling fractions of $f=0.25$ and 0.3 , respectively. The corresponding second band gap opens at $(0.410-0.513)(2 \pi c / a)$ and $(0.385-0.491)(2 \pi c / a)$, respectively. One might assume that the heterostructure interface between these two lattices could serve as a waveguide for EM waves. In fact, recent studies showed that waveguides could be created by removing either one or several rows of cylinders in photonic crystals, $, 1,3,5-10$ or by relative lattice displacements along the waveguide extension direction. ${ }^{21}$ These homostructure interfaces can support guided modes whose EM fields are localized in the lateral direction of the interface. To see whether localized guided modes are present in our heterostructure interface, we have calculated the band structure of photon modes with wave vectors along the waveguide. In this direction, the system is translationally invariant with a period of $a$. We do not find any localized guided mode in this heterostructure, namely, no state exists with its frequency within either the ground or higher band gaps. Furthermore, the band gap for guided modes is equal to the overlapping band gap of two lattices, within the accuracy of numerical calculations (about $1 \%)$.

To further verify the lack of localized interface states in these heterostructures, we solve the bulk band structure of the heterostructure, considering waves propagating along other directions, and calculate the photon DOS of the system. The DOS's are obtained by solving over 4000 points of Bloch wave vectors that are uniformly distributed in the first Brillouin zone of the original lattice. It is found that the first and second band gaps lie at the frequencies of $(0.228-$ $0.284)(2 \pi c / a)$ and $(0.412-0.492)(2 \pi c / a)$ for the heterostructure. The band gap of the heterostructure is just the overlap of the band gaps for the two composite crystals. No state is found within the overlapping band gap. This means that no localized interface state is created along any direction inside the heterostructure. We have considered other filling fractions, and found the same results; no localized interface state is present.

We then consider another heterostructure composed of two semi-infinite square lattices of air cylinders embedded in a homogeneous dielectric background with $n=3.6$, where also only the filling fraction is different for the two lattices. Here we use $f=0.6$ and 0.65 . For these crystals, there exists a significant band gap for both the TM and the TE modes in the low-frequency bands. The TM band gap lies at $(0.217-$ $0.240)(2 \pi c / a)$ and $(0.222-0.257)(2 \pi c / a)$, respectively, while the TE band gap opens at $(0.384-0.454)(2 \pi c / a)$ and $(0.404-0.477)(2 \pi c / a)$. Calculations of the band structure for the guided mode and the whole DOS along all directions in these heterostructures show the same result as in the heterostructure of dielectric cylinders. When all the cylinders are centered at the square lattice sites, no localized guided mode and interface state are present; the band gap is exactly equal to the overlap of the band gaps of the two composite crystals.

To understand more clearly why the simple heterostructures above do not support localized interface states and guided modes, we look at two extreme systems: One is the homostructure with $f_{1}=f_{2}$; the other has $f_{1}=0$ while $f_{2}$ $=f$, i.e., one semi-infinite crystal with a surface. The former structure is a perfect crystal and surely no interface state exists. The localized interface states or guided modes can be generated either by removing rows of cylinders or by relative longitudinal displacement of two lattices. In these cases, the line defect resembles somewhat the characteristic of a resonant cavity, which can support localized cavity eigenmodes inside the band gap. In the case of a 2D photonic crystal with a surface, recent theoretical and experimental studies ${ }^{12-14}$ showed that localized surface states could not be found for a crystal terminated by complete cylinders, yet, when the crystal was terminated with a surface layer of cylinders cut in half (hemicylinders), surface states could be observed. Our heterostructure considered above is essentially similar to the lattice with a surface terminated by complete cylinders; therefore it is no surprise that no localized interface state is present. Following this simple physical viewpoint, we could create localized interface states in more complex heterostructures, for example, structures where rows of cylinders at the 


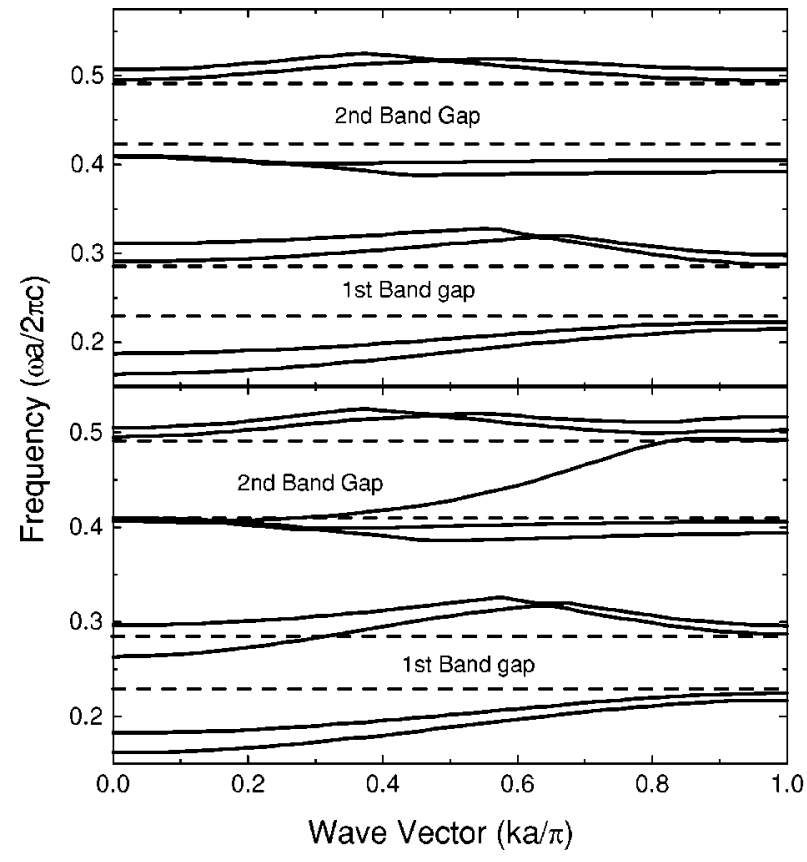

(a)

(b)

FIG. 2. Band structures for TM guided modes in two different heterostructures created by lattice displacement along (a) (01) direction, (b) (10) direction. The system is composed of dielectric cylinders in air with $f_{1}=0.3$ and $f_{2}=0.25$. Horizontal dashed lines represent edges of the overlapping band gap of two composite lattices. Modes inside the band gap are localized interface states.

interface are removed, or two lattices are slipped relative to each other along the interface direction, or two lattices are pulled closer to each other to allow cylinders at the interface to touch.

Indeed, things change greatly when we consider other kinds of more complex heterostructure, one created by slipping two semi-infinite lattices relative to each other along the (01) direction by half a lattice constant, and the other generated by increasing the interface separation by one lattice constant. The corresponding band structures of the guided mode for the system composed of dielectric cylinders in air are displayed in Figs. 2(a) and 2(b), respectively. Here, horizontal dashed lines represent the edges of the overlapping band gap of two composite lattices. Only several bands around the band gap are shown. The parameters of the system are $f_{1}$ $=0.3, f_{2}=0.25$, and $n=3.6$, and EM waves are assumed to be polarized in the TM mode. In the calculation, the supercell technique is also employed. The results for the system composed of air cylinders in a dielectric background are shown in Fig. 3 and Fig. 4 for the TM and TE modes, respectively. Here the other parameters are the same, $f_{1}$ $=0.6, f_{2}=0.65$, and $n=3.6$. In contrast with the simple heterostructure with no lattice displacement, we find in these complex heterostructures many guided modes inside the overlapping band gaps for two composite crystals, implying the existence of localized interface states. The exception occurs in the heterostructure corresponding to Fig. 2(a). These features are similar to those appearing in photonic crystal homostructures. ${ }^{1,3,5-10}$

We have investigated other filling fractions, and found

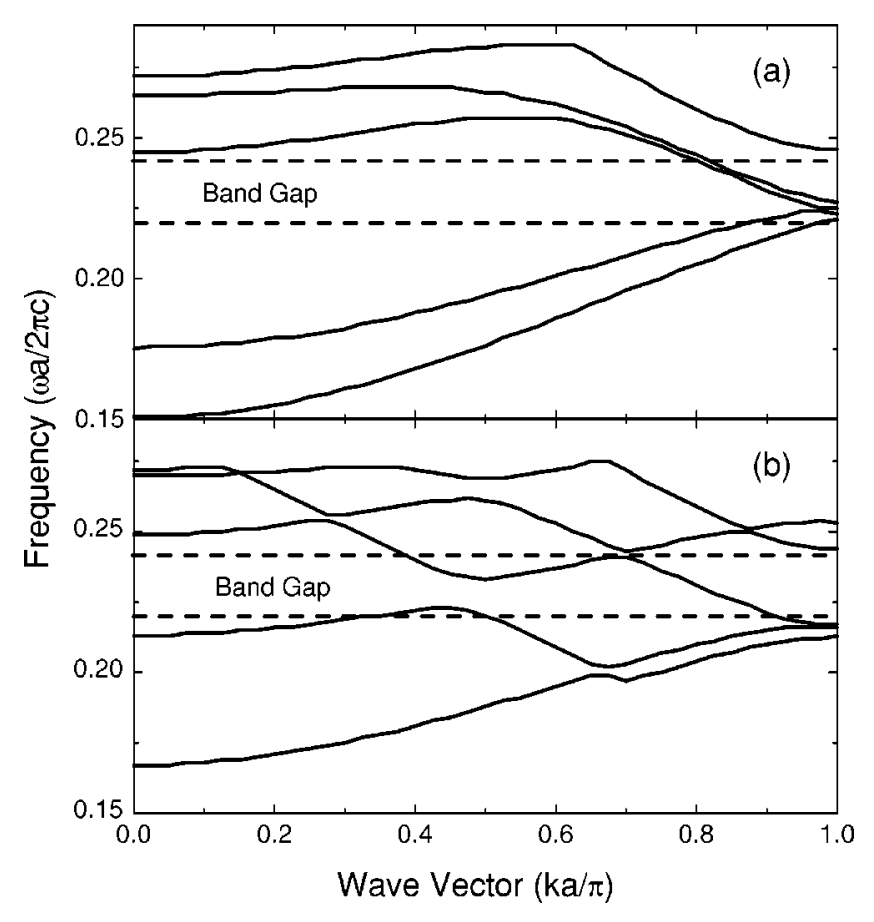

FIG. 3. Band structures for TM guided modes in two different heterostructures created by lattice displacement along (a) (01) direction, (b) (10) direction. The system is composed of air cylinders in dielectric with $f_{1}=0.6$ and $f_{2}=0.65$.

similar results. The creation of localized interface states in heterostructures with relative lattice slipping can be further understood as follows. In the case of perpendicular (10) slipping, the interface space is increased, and provides a wide enough potential well at the interface to lower the energy of

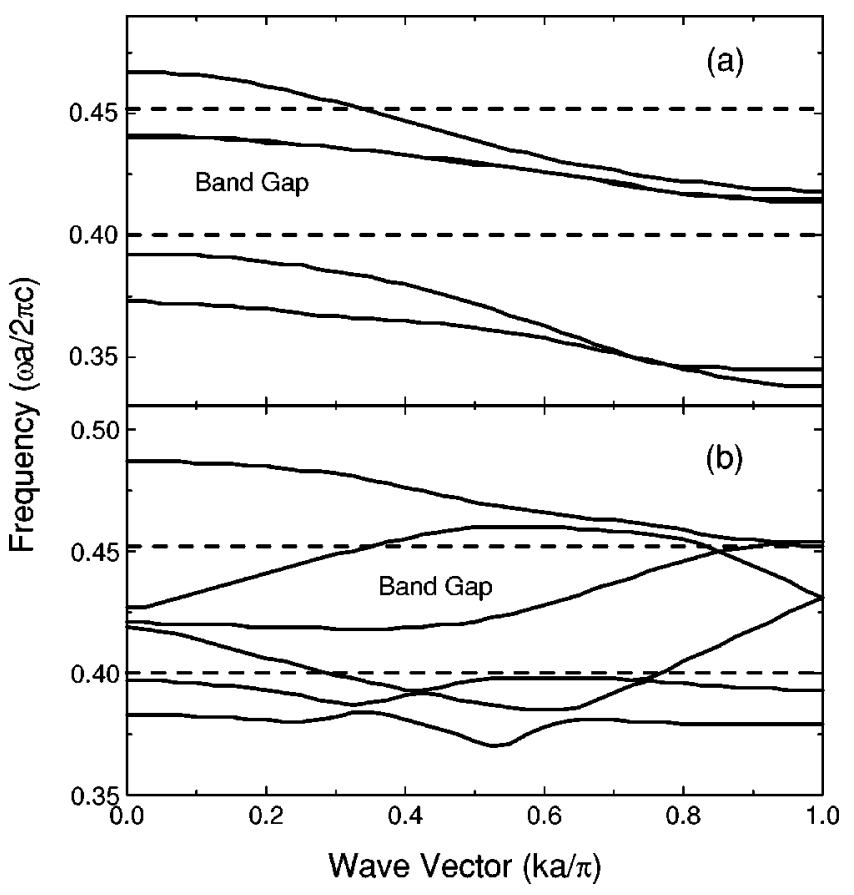

FIG. 4. Band structures for TE guided modes for heterostructures corresponding to those shown in Fig. 3. 
the ground state so that this energy falls inside the potential well. If the interface is too narrow, the energy of the ground eigenmode will lie beyond the potential well, and naturally no localized interface state is present. In the case of lateral (01) lattice slipping for a distance of $0.5 a$, one can find a cavitylike void surrounded by the three nearest cylinders around the interface. When the size of the cylinders is large enough, like the structures shown in Figs. 3(a) and 4(a) (nearly close packed), the cavity is closed enough to create a cavity mode, a localized interface state. In contrast, the cavity formed in the heterostructure shown in Fig. 2(a) is almost open at filling fractions $f=0.3$ and 0.25 ; therefore, no cavity mode is present. This can explain the different characteristics found in Figs. 2(a), 3(a), and 4(a). It is thus evident that the geometrical configuration of the cavity, its separation in space, and the configuration of the edge, play a key role in supporting localized interface states.

It might be interesting to make a comparison with conventional semiconductor heterostructures. ${ }^{22}$ In many semiconductor heterostructures, the atomic (ionic and electronic) interaction between two lattices will produce a deep potential well at the interface, and an electron gas is localized in this well. It is much easier to form a localized interface state than in photonic crystal heterostructures. The essential origin of this difference might be that the electromagnetic interaction in photonic crystals is long range (via EM wave radiation) and the localization take places through multireflection and multiscattering effects; however, in semiconductor heterostructure the ionic and electronic interactions are both short range and are strong enough to affect neighboring atoms. Therefore, it is more likely for electrons (with static mass) to localize inside some potential wells than for photons (no static mass) to localize in heterostructure interfaces.

In summary, we have studied simple heterostructures in 2D photonic crystals that are composed of two semi-infinite lattices with different filling fractions of cylinders. The plane-wave expansion method in combination with a supercell technique is employed to solve the photonic band structures and the DOS's of the heterostructures. Localized interface states are absent in simple heterostuructures with all cylinders lying in original lattice sites. Relative lattice displacements along the longitudinal and perpendicular directions can create the appropriate geometrical configuration of the cavity for resonant wave oscillation to produce localized interface states.

The authors gratefully acknowledge financial support from the National Natural Science Foundation of China, Hong Kong RGC Grant No. HKUST 6160/99P, and from the Alfred P. Sloan Foundation.
${ }^{1}$ J. D. Joannopoulos, R. D. Meade, and J. N. Winn, Photonic Crystals (Princeton University Press, Princeton, NJ, 1995).

${ }^{2}$ E. Yablonovitch, Phys. Rev. Lett. 58, 2059 (1987).

${ }^{3}$ J. D. Joannopoulos, P. R. Villeneuve, and S. Fan, Nature (London) 386, 143 (1997).

${ }^{4}$ Z. Y. Li, L. L. Lin, and Z. Q. Zhang, Phys. Rev. Lett. 84, 4341 (2000).

${ }^{5}$ A. Mekis, J. C. Chen, I. Kurland, S. Fan, P. R. Villeneuve, and J. D. Joannopoulos, Phys. Rev. Lett. 77, 3787 (1996).

${ }^{6}$ A. Mekis, S. Fan, and J. D. Joannopoulos, Phys. Rev. B 58, 4809 (1998).

${ }^{7}$ A. Chutinan and S. Noda, Appl. Phys. Lett. 75, 3739 (1999).

${ }^{8}$ S. Y. Lin, E. Chow, V. Hietala, P. R. Villeneuve, and J. D. Joannopoulos, Science 282, 274 (1998).

${ }^{9}$ J. C. Knight, J. Broeng, T. A. Birks, and P. St. J. Russell, Science 282, 1476 (1998).

${ }^{10}$ M. Tokushima, H. Kosaka, A. Tomita, and H. Yamada, Appl. Phys. Lett. 76, 952 (2000).

${ }^{11}$ J. E. G. J. Wijnhoven and W. L. Vos, Science 281, 802 (1998); Z.
Zhong, Y. Yin, B. Gates, and Y. Xia, Adv. Mater. 12, 206 (2000).

${ }^{12}$ W. M. Robertson et al., Opt. Lett. 18, 528 (1993).

${ }^{13}$ See Ref. 1, Chap. 5.

${ }^{14}$ F. Ramos-Mendieta and P. Halevi, Phys. Rev. B 59, 15112 (1999).

${ }^{15}$ Z. Y. Li, X. Zhang, and Z. Q. Zhang, Phys. Rev. B 61, 15738 (2000).

${ }^{16}$ K. M. Ho, C. T. Chan, and C. M. Soukoulis, Phys. Rev. Lett. 65, 3152 (1990).

${ }^{17}$ Z. Y. Li, J. Wang, and B. Y. Gu, Phys. Rev. B 58, 3721 (1998).

${ }^{18}$ D. Cassagne, C. Jouanin, and D. Bertho, Phys. Rev. B 53, 7134 (1996).

${ }^{19}$ M. Plihal and A. A. Maradudin, Phys. Rev. B 44, 8565 (1991).

${ }^{20}$ Z. Y. Li, B. Y. Gu, and G. Z. Yang, Phys. Rev. Lett. 81, 2574 (1998); Eur. Phys. J. B 11, 65 (1999).

${ }^{21}$ L. L. Lin and Z. Y. Li (unpublished).

${ }^{22}$ C. Kittel, Introduction to Solid State Physics (Wiley, New York, 1996), Chap. 19 